\title{
Facial Occlusion Reconstruction: Recovering Both the Global Structure and the Local Detailed Texture Components
}

\author{
Ching-Ting Tu and Jenn-Jier James Lien \\ Robotics Laboratory, Department of Computer Science and Information Engineering, \\ National Cheng Kung University, Tainan, Taiwan, R.O.C. \\ \{vida, jjlien\}@csie.ncku.edu.tw \\ http: //robotics.csie.ncku.edu.tw
}

\begin{abstract}
An automatic facial occlusion reconstruction system based upon a novel learning algorithm called the direct combined model (DCM) approach is presented. The system comprises two basic DCM modules, namely a shape reconstruction module and a texture reconstruction module. Each module models the occluded and non-occluded regions of the facial image in a single, combined eigenspace, thus preserving the correlations between the geometry of the facial features and the pixel grayvalues, respectively, in the two regions. As a result, when shape or texture information is available only for the nonoccluded region of the facial image, the optimal shape and texture of the occluded region can be reconstructed via a process of Bayesian inference within the respective eigenspaces. To enhance the quality of the reconstructed results, the shape reconstruction module is rendered robust to facial feature point labeling errors by suppressing the effects of biased noises. Furthermore, the texture reconstruction module recovers the texture of the occluded facial image by synthesizing the global texture image and the local detailed texture image. The experimental results demonstrate that compared to existing facial reconstruction systems, the reconstruction results obtained using the proposed DCM-based scheme are quantitatively closer to the ground truth.
\end{abstract}

Keywords: Facial reconstruction, facial synthesis, eigenspace, facial occlusion.

\section{Introduction}

The performance of automatic face recognition, facial expression analysis and facial pose estimation schemes is largely dependent upon the amount of information available in the input facial images. However, in real life, facial images are invariably occluded to a greater or lesser extent, and hence the performance of such schemes is inevitably degraded. It is necessary to develop the means to recover the occluded region(s) of the facial image such that the performance of these applications can be improved.

Saito et al. [14] proposed a method for removing eyeglasses and reconstructing the facial image by applying principal component analysis (PCA) to eigenspaces having 
no eyeglass information. Similarly, Park et al. [13] removed eyeglasses from facial images by repainting the pixels in the occluded region of the image with the grayvalues of the corresponding region of the mean facial image prior to the PCA reconstruction process. However, in both studies, the reconstruction process was performed based upon eigenspaces derived from the entire facial image rather than from the occluded and non-occluded regions, respectively. As a result, the two schemes are capable only of reconstructing facial images with long and thin occluded regions, e.g. occlusion by a pair of eyeglasses. If the major facial features, e.g. the eyes or the nose, are occluded, the schemes yield highly unpredictable and unrealistic reconstruction results. Furthermore, the reconstructed images tend to be notably blurred since both schemes use the Gaussian-distributed PCA process to model the facial images, whereas such images typically have a non-Gaussian distribution. To resolve this problem, the facial reconstruction systems presented in [7], [8] and [9] separated each facial image into its facial shape and facial texture, respectively, utilizing the face models introduced in [1], [3] and [15]. In contrast to the iterative facial reconstruction process presented in [9], Hwang et al. [7], [8] proposed a noniterative process for reconstructing the occluded region of an input face using facial shape and facial texture models. Each model consisted of one eigenspace and one sub-eigenspace, with the former containing the whole facial shape or texture information and the latter containing only the shape or texture information of the nonoccluded region. In the proposed approach, the shape or texture information of the non-occluded region was reconstructed via a linear combination of the sub-eigenspace and the corresponding weight vector. The whole facial image was then reconstructed by applying the same weight vector to the whole-face eigenspace. However, the significant characters of the two eigenspaces are different, and thus inherent variances between two different subjects may be suppressed if the same weight vectors are applied to both.

In contrast to the methods described above, apply a Gaussian distributed PCA process, the patch-based non-parametric sampling methods presented in [2], [5] and [11] synthesize facial images based upon local detailed features. In the psychological evaluations performed in [12], it was shown that facial features are correlated rather than independent. However, the localized characteristic of patch-based approaches results in a loss of information describing the overall geometric relationships between the individual facial features.

This paper proposes a learning-based facial occlusion reconstruction system comprising two DCM modules, namely a shape reconstruction module and a texture reconstruction module. Adopting a similar approach to that used in [3], the proposed system normalizes the texture image by warping the facial image to the mean-shape coordinates. The DCM approach used in the two modules facilitates the direct analysis of the geometric and grayvalue correlations of the occluded and nonoccluded regions of the face by coupling the shape and texture of the two regions within single shape and texture eigenspaces, respectively. Given the shape or texture of the non-occluded region of the face, the DCM modules enable the optimal shape or texture of the occluded region to be reconstructed even though the two regions of the 
face are modeled within a single eigenspace. In practice, the quality of the reconstructed facial shape is adversely effected by errors in the facial feature positions when labeling the features in the non-occluded region of the face. However, the shape reconstruction module developed in this study is specifically designed to tolerate such misalignments by taking account of these noise sources. Furthermore, the quality of the texture reconstruction results is enhanced by synthesizing the global texture image, i.e. a smooth texture image containing the global geometric facial structure, and a local detailed texture image, i.e. a difference image between the texture image and the corresponding global texture image.

\section{Direct Combined Model Algorithm}

The DCM algorithm assumes the existence of two related classes, i.e. $X \in R^{m}$ and $Y \in R^{n}$. Given an observable (or known) vector $x \in X$, such as the shape or pixel grayvalues of the non-occluded facial region, the objective of the DCM modules developed in this study is to estimate (i.e. recover) the corresponding unobservable (or unknown) vector $y \in Y$, i.e. the shape or pixel grayvalues of the occluded region, based on training datasets $X$ and $Y$. According to the maximum $a$ posterior (MAP) criterion, the optimal solution of the unknown $y$ can be obtained by maximizing the posterior probabilistic distribution $P(x \mid y, \theta)$, i.e.

$$
\hat{y}=\underset{y}{\arg \max } P(y \mid x, \theta)=\underset{y}{\arg \max } P(y \mid \theta) P(x \mid y, \theta),
$$

where $\theta$ denotes the model parameters, i.e. $\bar{x}, \bar{y}, C_{X Y}\left(\right.$ or $\left.C_{Y X}\right)$ and $C_{X X}\left(\right.$ or $\left.C_{Y Y}\right)$, in which $\bar{x}$ and $\bar{y}$ denote the mean vectors of classes $X$ and $Y$, respectively, $C_{X Y}$ (or $C_{Y X}$ ) is the cross-covariance matrix of $X$ and $Y$ (or $Y$ and $X$ ), and $C_{X X}$ (or $C_{Y Y}$ ) is the covariance matrix of $X$ (or $Y$ ), respectively. Assuming that $P(y \mid x, \theta)$ has a Gibbs distribution [4], [11], then

$$
P(y \mid x, \theta) \propto \exp \left\{-E_{G}(y, x, \theta)\right\},
$$

where $E_{G}(\bullet)$ is the Gibbs potential function, which describes the strength of the correlation between $\mathrm{x}$ and $\mathrm{y}$ based on the information contained within the training dataset and the model parameters $\theta$. Thus, Eq. (1) can be reformulated as an energy minimization problem of the form

$$
\hat{y}=\underset{y}{\arg \min } E_{G}(y, x, \theta) .
$$

In the reconstruction system presented in this study, the two training datasets, i.e. $X$ and $Y$, are modeled by combining them into a single joint Gaussian distribution using the PCA method. As a result, the combined training dataset, comprising $p$ training samples, can be represented as an $(m+n) \times p$ matrix, $\left[X^{\mathrm{T}} Y^{\mathrm{T}}\right]^{\mathrm{T}}$, in which each column corresponds to an unbiased, concatenated sample vector $\left[(x-\bar{x})^{\mathrm{T}}(y-\bar{y})\right]^{\mathrm{T}}$. 
Applying the singular value decomposition (SVD) process, the covariance matrix of the coupled training dataset can be expressed as

$$
\begin{aligned}
& {\left[\begin{array}{l}
X \\
Y
\end{array}\right]\left[\begin{array}{l}
X \\
Y
\end{array}\right]^{T}=\left[\begin{array}{ll}
C_{X X} & C_{X Y} \\
C_{Y X} & C_{Y Y}
\end{array}\right]=\left[\begin{array}{l}
U_{X} U_{\Delta} \\
U_{Y}
\end{array}\right]\left[\begin{array}{cc}
\Sigma_{K}^{2} & 0 \\
0 & \Sigma_{\Delta}^{2}
\end{array}\right]\left[\begin{array}{l}
U_{X} \\
U_{Y} U_{\Delta}
\end{array}\right]^{T},} \\
& =\left[\begin{array}{cc}
U_{X} \Sigma_{K}^{2} U_{X}^{T} & U_{X} \Sigma_{K}^{2} U_{Y}^{T} \\
U_{Y} \Sigma_{K}^{2} U_{X}^{T} & U_{Y} \Sigma_{K}^{2} U_{Y}^{T}
\end{array}\right]+\left[U_{\Delta} \Sigma_{\Delta}^{2} U_{\Delta}^{T}\right]
\end{aligned}
$$

where $U, \Sigma$ and $U_{\Delta}$ represent the combined eigenvector matrix, the combined eigenvalue matrix and the $m+n-K$ eigenvector matrix, respectively. According to the general properties of PCA, the linear combination of the first $K(K<<(m+n))$ eigenvectors, $\left[U_{X}{ }^{\mathrm{T}} U_{Y}{ }^{\mathrm{T}}\right]^{\mathrm{T}}$, sorted in descending order based on their corresponding eigenvalues, sufficiently represents all of the significant variances within the training dataset, and thus the remaining eigenvectors, $U_{\Delta}$, can be discarded. The resulting $K$ dimensional combined eigenspace, i.e. the DCM, can be used to reconstruct any new feature pair $\left(\hat{x}_{\mid w}, \hat{y}_{\mid w}\right)$ via a linear combination of the eigenspace and the corresponding $K$-dimensional weight vector, $w$, i.e.

$$
\left[\begin{array}{l}
x \\
y
\end{array}\right] \approx\left[\begin{array}{l}
\hat{x}_{\mid w} \\
\hat{y}_{\mid w}
\end{array}\right]=\left[\begin{array}{c}
U_{X} \\
U_{Y}
\end{array}\right] w+\left[\begin{array}{c}
\bar{x} \\
\bar{y}
\end{array}\right], \text { where } w=\left[\begin{array}{c}
U_{X} \\
U_{Y}
\end{array}\right]^{T}\left(\left[\begin{array}{l}
x \\
y
\end{array}\right]-\left[\begin{array}{c}
\bar{x} \\
\bar{y}
\end{array}\right]\right) .
$$

In the PCA technique, a set of orthogonal eigenvectors is obtained by minimizing the mean-square error (MSE) between the input data and the corresponding reconstruction result. In the current study, the minimum mean-square error (MMSE) is used as the criterion for the energy minimization problem given in Eq. (3). Hence, Eq. (3) can be expressed in terms of the expected posterior distribution $P(y \mid x, \theta)$ as follows:

$$
\hat{y}=\underset{y}{\arg \min } \int_{Y} y P(y \mid x, \theta) d Y=\underset{y, w}{\arg \min } \frac{1}{2}\left\|\left[\begin{array}{l}
x \\
y
\end{array}\right]-\left[\begin{array}{c}
\hat{x}_{\mid w} \\
\hat{y}_{\mid w}
\end{array}\right]\right\|^{2} .
$$

Substituting the parameters of the combined model, $\theta$, into Eq. (6) and applying the Penrose conditions method [6], Eq. (6) becomes

$$
\hat{y}=C_{Y X} C_{X X}^{-1}(x-\bar{x})=\bar{y}+U_{Y} U_{X}^{\dagger}(x-\bar{x}),
$$

where the singular matrix $\mathrm{U}_{\mathrm{X}}$ is the right inverse matrix of the non-square matrix $U_{X}$.

In contrast to the schemes presented in [7] and [16], in which the SVD algorithm is applied to approximate the inverse of this non-square matrix indirectly, the current study directly uses the following procedure to substitute the matrix inverse, $U_{X}^{\dagger}$. Since $U_{X} U_{X}^{\dagger}=I$ and the combined eigenspace, $\left[U_{X}^{\mathrm{T}}{ }^{\mathrm{T}} U_{Y}^{\mathrm{T}}\right]^{\mathrm{T}}$, is an orthonormal matrix, then $U_{X}{ }^{\mathrm{T}} U_{X}=I-U_{Y}^{\mathrm{T}} U_{Y}$. As a result, it can be inferred that: 


$$
\begin{aligned}
U_{X}^{T} I=U_{X}^{T} & \Leftrightarrow U_{X}^{T}\left(U_{X} U_{X}^{\dagger}\right)=U_{X}^{T} \\
& \Leftrightarrow\left(U_{X}^{T} U_{X}\right) U_{X}^{\dagger}=U_{X}^{T} \\
& \Leftrightarrow\left(I-U_{Y}^{T} U_{Y}\right) U_{X}^{\dagger}=U_{X}^{T}, \\
& \Leftrightarrow U_{Y}\left(I-U_{Y}^{T} U_{Y}\right) U_{X}^{\dagger}=U_{Y} U_{X}^{T} \\
& \Leftrightarrow\left(I-U_{Y} U_{Y}^{T}\right) U_{Y} U_{X}^{\dagger}=U_{Y} U_{X}^{T}
\end{aligned}
$$

in which the square matrix $\left(I-U_{Y} U_{Y}^{\mathrm{T}}\right)$ is invertible since classes $X$ and $Y$ are correlative. Hence, the $U_{Y} U_{X}^{\dagger}$ term in Eq. (7) can be replaced by $\left(I-U_{Y} U_{Y}^{T}\right)^{-1} U_{Y} U_{X}^{T}$, with the result that Eq. (7) becomes

$$
\hat{y}(x)=\bar{y}+\left(I-U_{Y} U_{Y}^{T}\right)^{-1} U_{Y} U_{X}^{T}(x-\bar{x}) .
$$

Here, the inverse of the residual covariance matrix, i.e. $\left(I-U_{Y} U_{Y}^{\mathrm{T}}\right)^{-1}$, is a normalization term which renders the correlation between $X$ and $Y$ insensitive to variances within each class. For example, if $X$ represents the grayvalues of the non-eye regions of the facial image and $Y$ represents the grayvalues of the eye region, the dynamic ranges of $X$ and $Y$ are clearly different. However, the normalization process renders the two ranges approximately equal to one another. In addition, the DCM algorithm assumes that classes $\mathrm{X}$ and $\mathrm{Y}$ are correlated. If this assumption is not made, i.e. $X$ and $Y$ are considered to be statistically uncorrelated, then $U_{Y} U_{X}{ }^{\mathrm{T}}$ becomes a zero matrix, i.e. $\hat{y}(x)=\bar{y}$.

\section{Reconstruction System}

The proposed reconstruction system for partially-occluded facial images is based on a joint Gaussian distribution assumption. However, in practice, the distribution of facial images actually has the form of a complicated manifold in a high-dimensional space, and thus it is inappropriate to model this distribution using a Gaussian distribution model. To resolve this problem, the current system separates the facial shape and texture of each image, rendering both facial properties more suitable for modeling using a Gaussian approach.

As shown in Fig. 1, the proposed facial occlusion reconstruction system comprises two separate DCM modules, namely the shape reconstruction module and the texture reconstruction module. In the training process, the facial feature points of each facial image in the training set are manually labeled to generate the corresponding facial shape, $S$, and the mean facial shape coordinates, $\bar{S}$, are then derived. Thereafter, each facial texture image with facial shape coordinates $S$ is warped to the mean facial shape $\bar{S}$ using a texture-warping transformation function $W[3]$ to generate the corresponding normalized texture image $T$. The resulting facial shapes $\{S\}$ and canonical textures $\{T\}$ of the training images are then used in the shape and texture modules, respectively, as described in the following. 


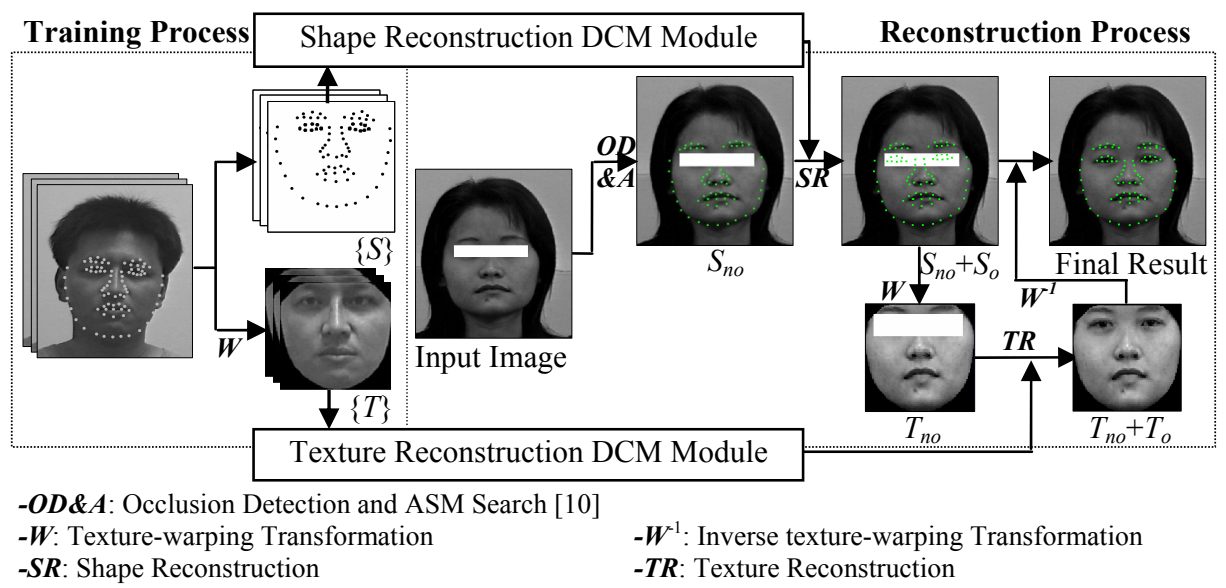

Fig. 1. Framework of partially-occluded facial image reconstruction system comprising shape reconstruction module and texture reconstruction module

In the shape reconstruction module, the occluded region of the input image and the facial shape of the non-occluded region, i.e. $S_{n o}$, are detected automatically using the method prescribed in [10]. The facial shape of the occluded region, i.e. $S_{o}$, is then reconstructed by the shape reconstruction DCM algorithm by applying a process of Bayesian inference to the facial shape of the non-occluded region to give the complete facial shape, $S$. Meanwhile, in the texture reconstruction module, the input texture image of the non-occluded region is warped from its original shape coordinates $S$ to the mean shape coordinates $\bar{S}$ using the transformation function $W$ to generate the corresponding normalized texture image of the non-occluded region, i.e. $T_{n o}$. The canonical facial texture of the occluded region, $T_{o}$, is then reconstructed from $T_{n o}$ using the texture reconstruction DCM algorithm. Finally, the complete canonical facial texture $T$ (i.e. $T_{n o}+T_{o}$ ) is warped from the mean facial shape coordinates $\bar{S}$ back to the original facial shape coordinates $S$ in order to generate the final reconstruction result.

The reconstruction process illustrated in Fig. 1 presents the particular case in which both eyes are occluded. However, due to the combined model approach, the reconstruction system developed in this study is capable of reconstructing frontalview facial images containing occluded regions in other facial features, such as the nose and the mouth, without modeling an additional combined model.

\subsection{Robustness of DCM Shape Reconstruction Module to Facial Feature Point Labeling Errors}

As shown in Fig.2, the DCM shape reconstruction module comprises a training process and a reconstruction process. In the training process, a $K$-dimensional shape eigenspace is constructed based upon a total of $p$ manually-labeled facial shapes $S$. The performance of the facial shape reconstruction module is highly dependent on the 


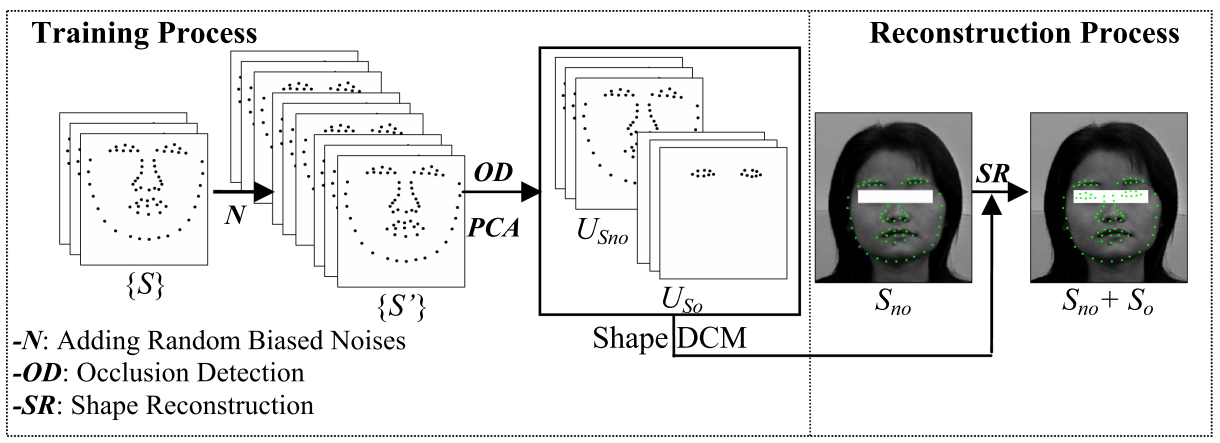

Fig. 2. Workflow of DCM shape reconstruction module

accuracy with which the individual facial feature points in the non-occluded region of the face image are identified. To improve the robustness of the shape reconstruction module, each training facial shape $S$ is added by $q$ number of random biased noises to generate a total of $q$ biased facial shapes $S$ '. The biased noise is randomly generated and is bounded by $\sigma_{c n}{ }^{2}\left(\sum_{K}{ }^{2}-\sigma_{c n}{ }^{2} I\right)^{-1}$ in accordance with the recommendations of the subspace sensitivity analyses presented in [6], where $\sum_{K}$ is the matrix of the first $K$ eigenvalues and $\sigma_{c n}{ }^{2}$ is the norm of the covariance matrix of the expected residual vector based on the $p$ training shape vectors $S$. Note that this residual vector is defined as the distance between the input facial shape and the corresponding reconstructed shape obtained using the $K$-dimensional shape eigenspace. A new facial shape eigenspace is then constructed based on the total of $p \times q$ facial shapes $S$ '.

Once the non-occluded and occluded regions of the input image have been detected and separated, the new facial shape eigenspace can be rearranged according to the combined eigenspace formula of the DCM algorithm, which is the non-occluded part, $X$, should be in the upper rows of the combined model, while the occluded part, $Y$, should be in the lower rows. Importantly, rearranging the eigenspace has no effect on the reconstruction result since exchanging any two row vectors in the combined eigenspace changes only their relative position in the eigenspace, i.e. the values of their elements are unchanged. Finally, the rearranged combined eigenspace is used to reconstruct the shape of the occluded region $S_{o}$ by replacing $x$ in Eq. (9) with $S_{n o}$.

\subsection{Recovery of Global Structure and Local Detailed Texture Components Using DCM Texture Reconstruction Module}

As shown in Fig. 3, the texture of an input image is reconstructed by integrating the global texture DCM and the local detailed texture DCM. The global texture image, i.e. $T^{g}$, is a smooth texture image containing the global geometric facial structure, while the local detailed texture image, i.e. $T$, represents the difference between $T^{g}$ and the normalized texture image $T$, and contains the subtle details of the facial texture. The objective function of the DCM texture reconstruction module can be formulated as

$$
\begin{aligned}
& T_{o}^{g}=\arg \max P\left(T_{n o}^{g} \mid T_{o}^{g}, \theta\right) P\left(T_{o}^{g}, \theta\right) \\
& T_{o}^{l}=\arg \max P\left(T_{n o}^{l} \mid T_{o}^{l}, \theta\right) P\left(T_{o}^{l}, \theta\right)
\end{aligned},
$$




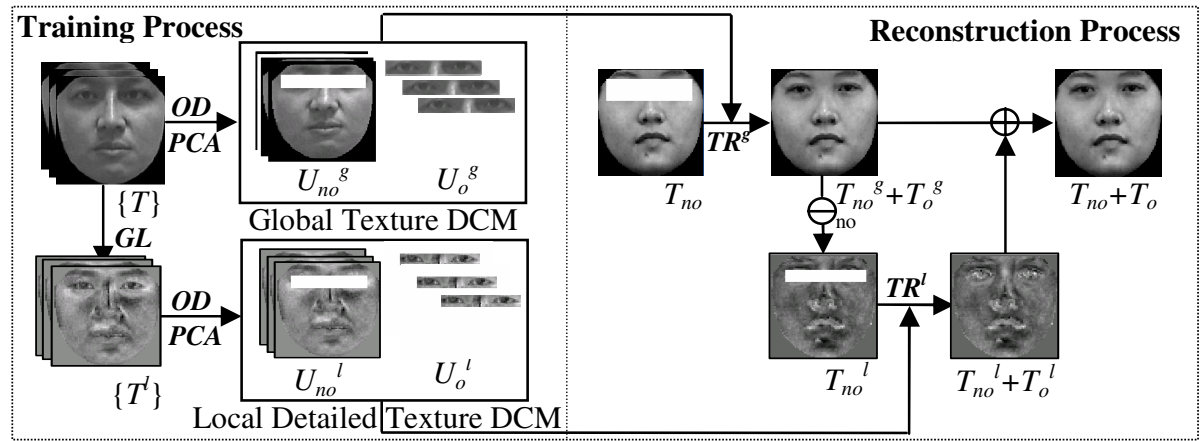

- GL: Generating Local Detailed Texture Image

- $\ominus_{\text {no }}$ : Image Subtraction in Non-occluded Region

- $\boldsymbol{T R}^{g}$ : Global Texture Reconstruction

- OD: Occlusion Detection

- $\bigoplus$ : Image Addition

- $\boldsymbol{T R}^{l}$ : Local Detailed Texture Reconstruction

Fig. 3. Workflow of DCM texture reconstruction module

where $T_{n o}{ }^{g}$ and $T_{n o}{ }^{l}$ are the global and local detailed texture components of $T_{n o}$, respectively, and $T_{o}{ }^{g}$ and $T_{o}{ }^{l}$ are the global and local detailed texture components of $T_{o}$, respectively.

In the training process, the texture image training dataset, $\{T\}$, is used to construct a $K$-dimensional global texture eigenspace. The local detailed texture images of the training dataset $\{T\}$ are then calculated and used to construct the local detailed texture eigenspace. Each local detailed texture image is derived by calculating the difference between its texture image, $T$, and the corresponding global texture image, $T^{g}$. In the reconstruction process, the texture of the occluded region $T_{o}$ is inferred via the following procedure:

1. According to the occluded region and the non-occluded region of the input texture image, the global eigenspace and the local detailed eigenspace are rearranged using the combined eigenspace formula of DCM given in Eq. (4).

2. Replacing $x$ in Eq. (9) with $T_{\text {no }}$, the global texture of the occluded region, i.e. $T_{\mathrm{o}}{ }^{\mathrm{g}}$, is reconstructed using the global texture DCM.

3. The image $T$, , which contains the texture of the non-occluded region $T_{n o}$ and the current reconstruction result $T_{o}^{g}$, is projected onto the $K$-dimensional global texture eigenspace, and the corresponding projection weight is then used to reconstruct $T$ ". The local detailed texture components of the non-occluded region are then extracted by calculating $T_{n o}{ }^{l}=T^{\prime \prime}-T^{\prime}$.

4. Replacing $x$ in Eq. (9) with $T_{n o}{ }^{l}$, the local detailed texture of the occluded region $T_{o}^{l}$ is reconstructed using the local detailed DCM.

5. The final texture result is obtained by synthesizing $T_{n o}$ with the reconstruction results $T_{o}{ }^{g}$ and $T_{o}$, i.e. $T=T_{n o}+T_{o}{ }^{g}+T_{o}^{l}$.

\section{Experimental Results}

The performance of the proposed reconstruction system was evaluated by performing a series of experimental trials using training and testing databases comprising 205 and 
60 facial images, respectively. The images were acquired using a commercial digital camera at different times, in various indoor environments. Eighty-four facial feature points were manually labeled on each training and testing facial image to represent the ground truth of the facial shape. Specific facial feature regions of the testing images were then occluded manually.

Figure 4 presents representative examples of the reconstruction results obtained using the proposed method for input images with a variety of occlusion conditions. Figures 4(a) and 4(b) show the occluded facial images and the original facial images, respectively. Figure 4(c) presents the reconstruction results obtained using the shape and global texture DCMs. Meanwhile, Fig. 4(d) presents the reconstruction results obtained when the texture is reconstructed using not only the global texture DCM, but also the local detailed texture DSM. Comparing the images presented in Fig. 4(d) with

(a)
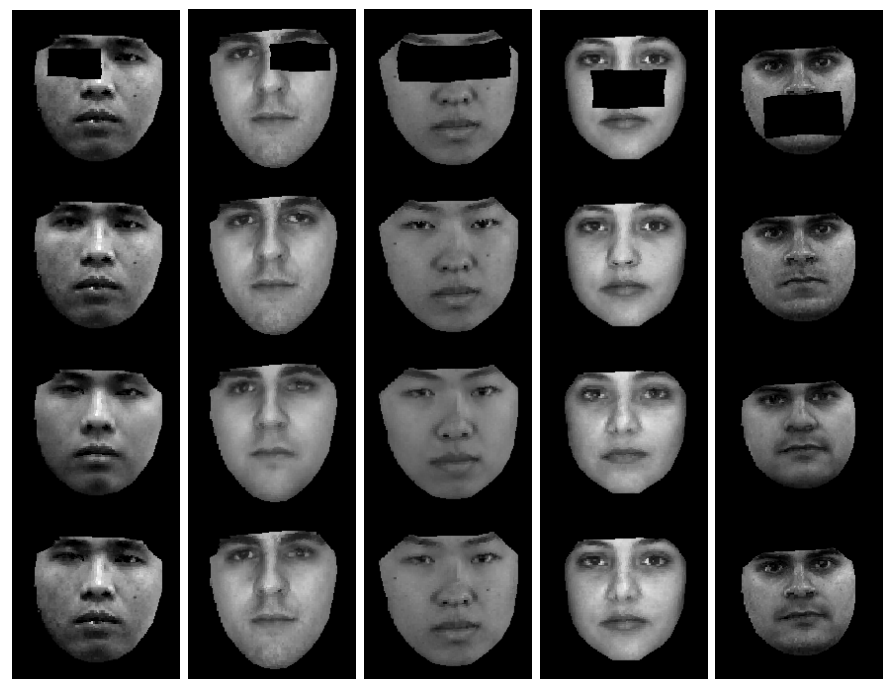

Fig. 4. Reconstruction results obtained using DCM method: (a) occluded facial images, (b) original facial images, (c) reconstructed facial images using global texture DCM only, and (d) final reconstruction results using both global texture DCM and local texture DCM

Table 1. Average and standard deviation of facial shape and facial texture reconstruction errors for images in testing database with different levels of occlusion. Note that the occlusion rate data indicate the ratio of the occluded area to the non-occluded area in the facial image; Ave: Average; Std. Dev: Standard deviation of errors.

\begin{tabular}{lccccc}
\hline Facial & \multicolumn{2}{c}{ Ave. Error (Pixel/Grayvalues) } & \multicolumn{2}{c}{ Std. Dev. (Pixel/Grayvalues) } & \multicolumn{2}{l}{$\begin{array}{l}\text { Occlusion } \\
\text { features }\end{array}$} & Shape & Texture & Shape & Texture & Rate \\
\hline Left Eye & 1.2 & 6.6 & 1.1 & 1.7 & $10 \%$ \\
Right Eye & 1.3 & 6.5 & 1.0 & 1.8 & $10 \%$ \\
Both Eye & 1.4 & 8.0 & 1.7 & 3.6 & $24 \%$ \\
Nose & 1.0 & 7.2 & 1.4 & 3.0 & $16 \%$ \\
Mouth & 1.6 & 6.8 & 1.5 & 3.2 & $20 \%$ \\
\hline
\end{tabular}


(a)

(b)

(c)

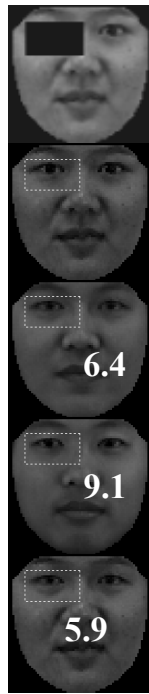

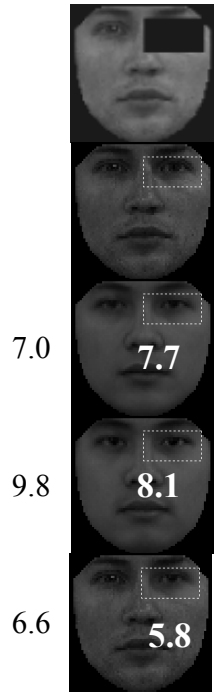
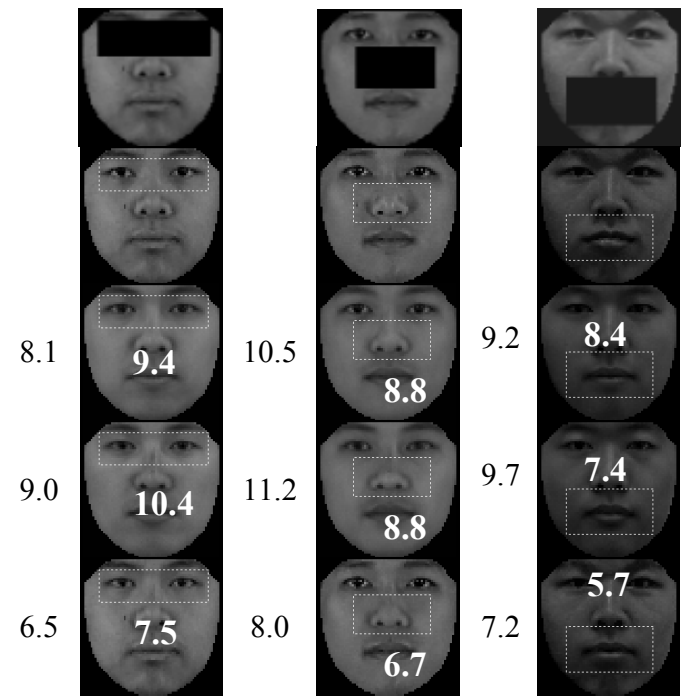

8.7

Fig. 5. Reconstruction results: (a) occluded facial images, (b) original facial images, (c) reconstructed texture images using method presented in [13], (d) reconstructed texture images using method presented in [8], and (e) reconstructed texture images using current DCM method. The digits within the images represent the average grayvalue evaluation error of the corresponding pixels in the original non-occluded image, while the digits in the columns next to these images represent the average grayvalue error over all of the images in the test database. Note that each facial texture image has a size of $100 * 100$ pixels.

those presented in Fig. 4(b), it can be seen that the use of the two texture DCMs yields a highly accurate reconstruction of the original facial image. Table 1 presents the average reconstructed shape and texture errors computed over all the images in the testing database. In general, the results show that the magnitudes of both errors increase as the level of occlusion increases or as the geometrical complexity of the occluded facial feature increases. Figure 5 compares the reconstruction results obtained using the proposed DCM method with those obtained using the occlusion recovery schemes presented in [8] and [13], respectively. The data presented within the reconstructed images indicate the average difference between the grayvalues of the pixels in the restored region of the reconstructed image and the grayvalues of the corresponding pixels in the original non-occluded image, while the data in the columns next to these images indicate the average grayvalue error of the restored pixels in the occluded region computed over all 60 texture images within the test database. Overall, the results demonstrate that the images reconstructed using the current DCM-based method are closer to the original un-occluded facial images than those obtained using the schemes presented in [8] or [13].

\section{Conclusions}

This study has presented an automatic facial occlusion reconstruction system comprising two DCM-based modules, namely a shape reconstruction module and a 
texture reconstruction module, respectively. The experimental results have demonstrated that the images reconstructed by the proposed system closely resemble the original non-occluded images. The enhanced reconstruction performance of the proposed system arises as a result of its robustness toward misalignments of the facial features when constructing the facial shape and its ability to recover both the global structure and the local detailed facial texture components of the input image. Unlike PCA-based methods, the DCM-based system presented in this study provides the ability to reconstruct the occluded region of an input image directly from an image of the non-occluded region even though they are initially combined in a single eigenspace. Overall, the experimental results indicate that the DCM-based facial occlusion reconstruction system presented in this study represents a promising means of enhancing the performance of existing automatic face recognition, facial expression recognition, and facial pose estimation applications.

\section{References}

1. Blanz, V., Romdhani, S., Vetter, T.: Face Identification across Different Poses and Illuminations with a 3D Morphable Model. In: IEEE Intl. Conf. on FG, pp. 202-207 (2002)

2. Chen, H., Xu, Y.Q., Shum, H.Y., Zhu, S.C., Zhen, N.N.: Example-Based Facial Sketch Generation with Non-Parametric Sampling. In: Proceedings of ICCV, pp. 433-438 (2001)

3. Cootes, T.F., Taylor, C.J.: Statistical Models of Appearance for Computer Vision. Technical Report, Univ. of Manchester (2000)

4. Fisker, R.: Making Deformable Template Models Operational. PhD Thesis, Informatics and Mathematical Modelling, Technical University of Denmark (2000)

5. Freeman, W.T., Pasztor, E.C.: Learning Low-Level Vision. In: ICCV, pp. 1182-1189 (1999)

6. Golub, G.H., Van Loan, C.F.: Matrix Computations, 3rd edn. Johns Hopkins University Press, Baltimore (1996)

7. Hwang, B.W., Blanz, V., Vetter, T., Lee, S.W.: Face Reconstruction from a Small Number of Feature Points. In: Proceedings of ICPR, pp. 842-845 (2000)

8. Hwang, B.W., Lee, S.W.: Reconstruction of Partially Damaged Face Images Based on a Morphable Face Model. IEEE Trans. on PAMI 25(3), 365-372 (2003)

9. Jones, M.J., Poggio, T.: Multidimensional Morphable Models: A Framework for Representing and Matching Object Classes. IJCV 29(2), 107-131 (1998)

10. Lanitis, A.: Person Identification from Heavily Occluded Face Images. In: Handschuh, H., Hasan, M.A. (eds.) SAC 2004. LNCS, vol. 3357, pp. 5-9. Springer, Heidelberg (2004)

11. Liu, C., Shum, H.Y., Zhang, C.S.: A Two-Step Approach to Hallucinating Faces: Global Parametric Model and Local Nonparametric Model. In: Proc. of CVPR, pp. 192-198 (2001)

12. Mo, Z., Lewis, J.P., Neumann, U.: Face Inpainting with Local Linear Representations. In: Proceedings of British Machine Vision Conference, vol. 1, pp. 347-356 (2004)

13. Park, J.S., Oh, Y.H., Ahn, S.C., Lee, S.W.: Glasses Removal from Facial Image Using Recursive PCA Reconstruction. IEEE Trans. on PAMI 27(5), 805-811 (2005)

14. Saito, Y., Kenmochi, Y., Kotani, K.: Estimation of Eyeglassless Facial Images Using Principal Component Analysis. In: Proceedings of ICIP, vol. 4, pp. 197-201 (1999)

15. Vetter, T., Poggio, T.: Linear Object Classes and Image Synthesis from a Single Example Image. IEEE Trans. on PAMI 19(7), 733-742 (1997)

16. Wu, C., Liu, C., Shum, H.Y., Xu, Y.Q., Zhang, Z.: Automatic Eyeglasses Removal from Face Images. IEEE Trans. PAMI 26, 322-336 (2004) 